\title{
Spin and orbital ordering in bilayer $\mathrm{Sr}_{3} \mathrm{Cr}_{2} \mathrm{O}_{7}$
}

\author{
Armando A. Aligia ${ }^{1,2, *}$ and Christian Helman ${ }^{1}$ \\ ${ }^{1}$ Centro Atómico Bariloche and Instituto Balseiro, CNEA, 8400 S. C. de Bariloche, Argentina \\ ${ }^{2}$ Consejo Nacional de Investigaciones Científicas y Técnicas, 8400 S. C. de Bariloche, Argentina
}

(Received 18 December 2018; revised manuscript received 14 March 2019; published 28 May 2019)

\begin{abstract}
Using maximally localized Wannier functions obtained from density functional theory calculations, we derive an effective Hubbard Hamiltonian for a bilayer of $\mathrm{Sr}_{3} \mathrm{Cr}_{2} \mathrm{O}_{7}$, the $n=2$ member of the Ruddlesden-Popper $\mathrm{Sr}_{n+1} \mathrm{Cr}_{n} \mathrm{O}_{3 n+1}$ system. The model consists of effective $t_{2 g}$ orbitals of $\mathrm{Cr}$ in two square lattices, one above the other. The model is further reduced at low energies and two electrons per site to an effective Kugel-Khomskii Hamiltonian that describes interacting spins 1 and pseudospins $1 / 2$ at each site describing spin and orbital degrees of freedom, respectively. We solve this Hamiltonian at zero temperature using pseudospin bond operators and spin waves. Our results confirm a previous experimental and theoretical study that proposes spin ordering is antiferromagnetic in the planes and ferromagnetic between planes, while pseudospins form vertical singlets, although the interplane separation is larger than the nearest-neighbor distance in the plane. We explain the physics behind this rather unexpected behavior.
\end{abstract}

DOI: 10.1103/PhysRevB.99.195150

\section{INTRODUCTION}

Some decades ago, Kugel and Khomskii studied theoretically the interplay between orbital and spin degrees of freedom in compounds like $\mathrm{KCuF}_{3}$ and $\mathrm{K}_{2} \mathrm{CuF}_{4}$ [1]. They showed that in these compounds the $e_{g}$ orbital degrees of freedom (leaving the hole in the $3 d^{9}$ configuration of $\mathrm{Cu}$ in the orbital with symmetry being either $x^{2}-y^{2}$ or $3 z^{2}-r^{2}$ ) can be described by a pseudospin, and these pseudospins interact with each other and with the spins of the $\mathrm{Cu}$ ions in such a way that the preferred ordering is antiferromagnetic for the pseudospins and the spin ordering is ferromagnetic in the $a b$ plane and antiferromagnetic in the $c$ direction. The staggered ordering of the orbitals leads to a staggering of quadrupolar distorted $\mathrm{CuF}_{4}$ units in the $a b$ planes, as expected from any electron-phonon interaction [2].

The interest in systems with spin and orbital degrees of freedom has risen over the years (see, for example, Refs. [2-21]). A rich physics has been observed in the $n=2$ members of the Ruddlesden-Popper series of the form $A_{n+1} B_{n} C_{3 n+1}$, where $B$ denotes transition-metal atoms that form two square lattices in the $x y$ plane, one displaced with respect to the other in the $z$ direction $[5,12,15,21]$. In the layered colossal magnetoresistance manganite $\mathrm{La}_{2-2 x} \mathrm{Sr}_{1+2 x} \mathrm{Mn}_{2} \mathrm{O}_{7}$, different spin and orbital orderings are observed as $x$ is varied, indicating that $e_{g}$ orbital polarization is the driving force behind spin ordering [5]. Particularly interesting is $\mathrm{K}_{3} \mathrm{Cr}_{2} \mathrm{O}_{7}$, where distortions reveal antiferromagnetic orbital ordering, while the system presents a spin gap due to spin dimers in the $z$ direction [12]. This is an exotic ordering involving spins and pseudospins both of magnitude 1/2. In $\mathrm{Sr}_{3} \mathrm{Ru}_{2} \mathrm{O}_{7}$, an applied magnetic field induces domains with distorted lattice parameters likely related to orbital ordering [15].

\footnotetext{
*aligia@cab.cnea.gov.ar
}

Recently, the compound $\mathrm{Sr}_{3} \mathrm{Cr}_{2} \mathrm{O}_{7}$ was studied by several experimental and theoretical techniques [21]. The resistivity and specific heat measurements indicate that the system is insulating. The calculations using density functional theory (DFT) with local-density approximation (LDA) $+U$ approximation indicate that occupancy of $\mathrm{Cr}$ is consistent with an oxidation state of $\mathrm{Cr}^{+4}$ (two electrons in the $3 d$ shell) and there is an orbital degeneracy between $d_{x y}^{1} d_{x z}^{1}$ and $d_{x y}^{1} d_{y z}^{1}$. Hund's rules favor a total spin $S=1$. There is a magnetic transition at $210 \mathrm{~K}$ with a huge total entropy change near $R \ln (6)$ indicating a simultaneous spin and orbital ordering. The magnetic structure observed by neutron diffraction is consistent with the DFT results and corresponds to antiferromagnetic alignment between nearest neighbors in the plane and ferromagnetic alignment between planes. Orbital ordering is usually not detected in DFT with LDA and derived potentials due to the difficulties of these techniques to obtain orbital polarization $[2,22]$. $\mathrm{LDA}+U$ is able to capture orbital ordering [2] but not singlet ordering because it is based on a single Slater determinant. The LDA $+U$ calculations of Ref. [21] do not detect any orbital ordering. Nevertheless, the absence of observable distortions that accompany the orbital ordering (as in $\mathrm{KCuF}_{3}$ and $\mathrm{K}_{2} \mathrm{CuF}_{4}$ mentioned above) is consistent with the formation of vertical singlet orbital dimers, so that quantum fluctuations destroy long-range pseudospin ordering. This spin/pseudospin state has some analogy to the case of $\mathrm{K}_{3} \mathrm{Cu}_{2} \mathrm{O}_{7}$ (with both spin and pseudospin $S=1 / 2$ ) mentioned in the previous paragraph with spin and pseudospin (orbital) degrees of freedom interchanged.

Jeanneau et al. also derived a Kugel-Khomskii Hamiltonian from a multiband Hubbard model containing the relevant orbitals of $\mathrm{Cr}$ and $\mathrm{O}$ [21]. Solving this Hamiltonian by Lanczos in a cluster containing eight sites, they found that, effectively, the ground state corresponds to the observed magnetic ordering and vertical pseudospin singlets if $t_{z}^{\prime} / t_{p}^{\prime}>0.85$, where $t_{z}^{\prime}\left(t_{p}^{\prime}\right)$ is the hopping between nearest- 
neighbor $\mathrm{Cr}$ and $\mathrm{O}$ orbitals perpendicular to (in) the $\mathrm{CrO}_{2}$ planes. Since $t_{z}^{\prime}$ is expected to be smaller than $t_{p}^{\prime}$ because of the larger distances in the plane and the size of the cluster is very small, further theoretical work is necessary to confirm that the proposed exotic spin and orbital ordering is, in fact, the ground state. Note that for a spin- $1 / 2$ Heisenberg model in a bilayer system (our bilayer system for only pseudospins, i.e., $S=0$ ) with interplane interaction $J$ and vertical interaction $J^{\prime}$, the quantum phase transition from a Néel ordered phase to the quantum disordered dimer-singlet phase takes place for $J^{\prime} / J \approx 2.522$ [23,24], and for the three-dimensional extension with two cubic sublattices the transition moves to $J^{\prime} / J \approx 4.84$ [25]. Therefore, naively, one would expect a phase with longrange pseudospin ordering in $\mathrm{Sr}_{3} \mathrm{Cr}_{2} \mathrm{O}_{7}$ for $t_{z}^{\prime} \sim t_{p}^{\prime}$.

In this work we first construct a tight-binding model for effective $t_{2 g}$ orbitals at the $\mathrm{Cr}$ sites, using maximally localized Wannier functions (MLWFs), and add to it the on-site interactions $[9,26]$. This leads to a three-band Hubbard model. This starting approach is similar to that followed by Ogura et al. to study the possible occurrence of superconductivity in hole-doped $\mathrm{Sr}_{3} \mathrm{Cr}_{2} \mathrm{O}_{7}$ and $\mathrm{Sr}_{3} \mathrm{Mo}_{2} \mathrm{O}_{7}$ [27]. These effective $t_{2 g}$ orbitals are not pure $\mathrm{Cr} 3 d$ orbitals of $x y, x z$, and $y z$ symmetry but contain an important admixture with $\mathrm{O}$ orbitals. Next, we derive a Kugel-Khomskii Hamiltonian for the low-energy subspace of two electrons per site by degenerate perturbation theory in the hopping terms. We explain the meaning of the different terms and the expected physics. Finally, this Hamiltonian is solved for the infinite system, using a combination of bond operators and spin waves. For the resulting parameters we find that the state of singlet dimers and the spin ordering proposed in Ref. [21] is, in fact, the ground state. To destabilize it, one would need to reduce the hopping $t_{z}^{\prime}$ mentioned above by a factor close to $1 / 2$.

This paper is organized as follows. In Sec. II we describe the atomic and electronic structures. Section III describes the $a b$ initio method used to obtain the effective hoppings and onsite energies used in Sec. IV to derive an effective multiband model for the system. In Sec. V we use this model to derive an effective Kugel-Khomskii Hamiltonian to describe the spin and orbital degrees of freedom of the model. This model is solved in Sec. VI using a generalized spin-wave theory. Section VII contains a summary and discussion.

\section{THE SYSTEM}

As a starting point we calculate the electronic structure of the $\mathrm{Sr}_{3} \mathrm{Cr}_{2} \mathrm{O}_{7}$ system within the framework of DFT. The structure has $I 4 / \mathrm{mmm}$ symmetry, which is tetragonal with lattice parameters $a=3.796 \AA$ and $c=19.846 \AA$ [21], and we include the asymmetric deformation of the $\mathrm{CrO}_{6}$ octahedra as reported in Ref. [21]. We distinguish three types of $\mathrm{O}$ atoms according to their bonding role. As shown in Fig. 1, the structure is a stacking of $\mathrm{CrO}_{2}$ layers and $\mathrm{SrO}$ layers. We label as $\mathrm{O}_{1}$ the $\mathrm{O}$ atoms inside the $\mathrm{CrO}_{2}$ layers, $\mathrm{O}_{2}$ denotes the $\mathrm{O}$ atoms in the $\mathrm{SrO}$ layers between two $\mathrm{CrO}_{2}$ layers, and $\mathrm{O}_{3}$ refers to the $\mathrm{O}$ atoms of the $\mathrm{SrO}$ layers that lie between another $\mathrm{Sr}-\mathrm{O}_{3}$ layer and a $\mathrm{CrO}_{2}$ one. Two consecutive $\mathrm{Sr}-\mathrm{O}_{3}$ layers are displaced by a vector $(a / 2, a / 2, d)$, where $d$ is the interlayer $\mathrm{Sr}_{-} \mathrm{O}_{3}$ distance, as can be seen in the small scheme on the right of Fig. 1. In the $\mathrm{CrO}_{2}$ layers, both $\mathrm{O}_{1}$ and $\mathrm{Cr}$ atoms

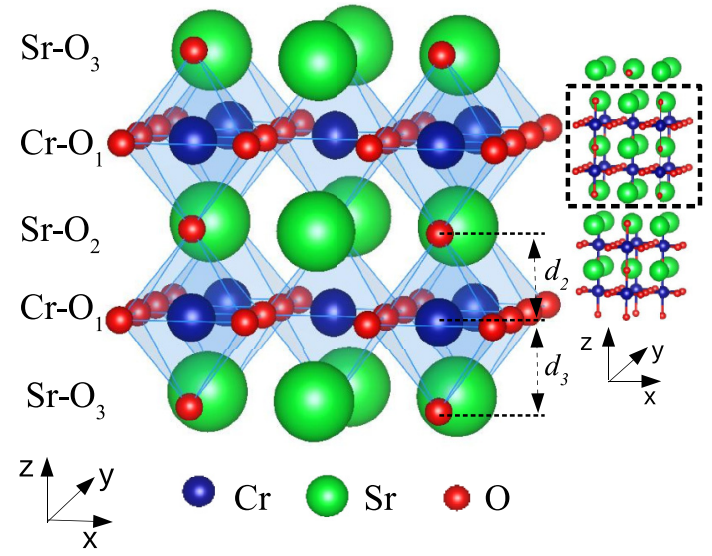

FIG. 1. Tetragonal structure of $\mathrm{Sr}_{3} \mathrm{Cr}_{2} \mathrm{O}_{7}$. The stacking is made of three types of layers. The unit cell (shown on the right) contains two blocks of five layers shown on the left; the second is displaced in the $x, y$ direction by $(a / 2, a / 2)$ with respect to the first one. $d_{2}$ and $d_{3}$ denote the distances $\mathrm{Cr}-\mathrm{O}_{2}$ and $\mathrm{Cr}-\mathrm{O}_{3}$, respectively. The ratio $d_{3} / d_{2}=1.016[21]$.

form a square lattice. Each $\mathrm{Cr}$ atom has four nearest neighbors that are $\mathrm{O}_{1}$ atoms in the $x, y$ plane and two more $\mathrm{O}$ neighbors in the $z$ direction; the one between $\mathrm{CrO}_{2}$ layers is type $\mathrm{O}_{2}$, and the other is type $\mathrm{O}_{3}$. Note that the distances between $\mathrm{Cr}$ and $\mathrm{O}_{2}$ and between $\mathrm{Cr}$ and $\mathrm{O}_{3}$ are different, as shown in Fig. 1 .

Since we are looking for the hopping parameters, the DFT calculations are performed as spin unpolarized but using both cell parameters and atomic coordinates obtained from the spin-polarized case. The band structure calculations are done using the WIEN2K code [28], with precision parameters $R_{M T} K_{\max }=7$, which reads as the product between the smallest muffin-tin sphere radius and the plane-wave cutoff $K_{\max }$. The Brillouin zone was sampled with a regular mesh containing 800 irreducible points, and we use the generalized gradient approximation for the potential of the exchange correlation.

The band structure and the atom-projected density of states are shown in Fig. 2, where band character is emphasized by color; red means a strong $\mathrm{Cr}$ component, and blue stands for the $\mathrm{O}$ component. The electronic structure close to the Fermi energy is dominated by $\mathrm{Cr} d$ states, which are split by the tetragonal component of the crystal field into $t_{2 g}$ and $e_{g}$ states. The $\mathrm{Cr} t_{2 g}$ states are the partially filled states and share a peak with $\mathrm{O}_{3}$ at $-0.7 \mathrm{eV}$; they also hybridize with in-plane $\mathrm{O}_{1}$ atoms. Above the Fermi level, the states of $\mathrm{Cr}$ share a peak with $\mathrm{O}_{2}$ at $0.09 \mathrm{eV}$. These characteristics in the DOS expose the bridging character of the $\mathrm{O}_{2}$ between the next $\mathrm{CrO}_{2}$ layers. The band structure presents a region around the Fermi level, where the $t_{2 g}$ states of the $\mathrm{Cr}$ prevail. The $e_{g}$-type orbitals are located at $0.9 \mathrm{eV}$ above the Fermi level.

Note that the band structure predicts a metallic state, while, actually, the compound is an insulator [21]. This is corrected when the interactions are included, as we do in Sec. IV.

\section{HOPPING PARAMETERS FROM MAXIMALLY LOCALIZED WANNIER FUNCTIONS}

The maximally localized Wannier function (MLWF) approach provides a physically intuitive and also rigorous 


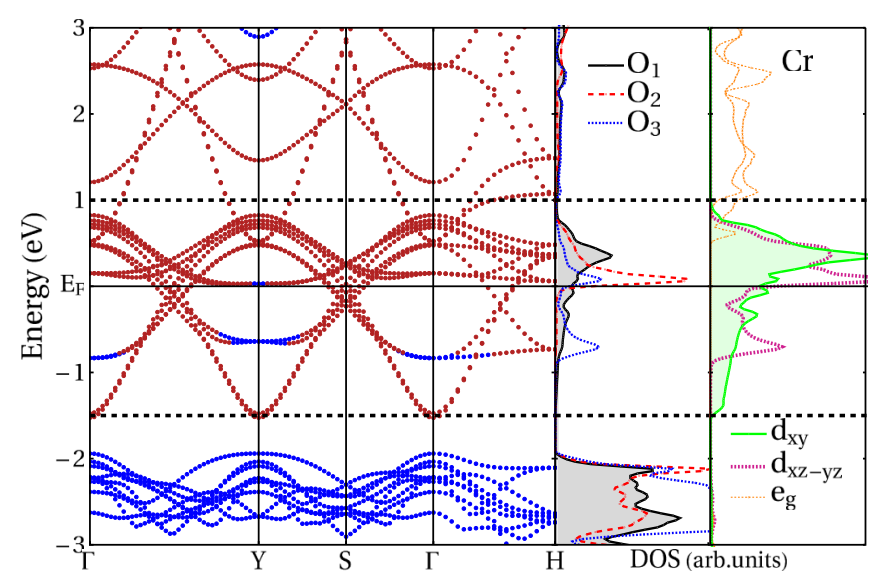

FIG. 2. Spin-unpolarized band structure of $\mathrm{Sr}_{3} \mathrm{Cr}_{2} \mathrm{O}_{7}$ (left panel) along with the atom-projected density of states (right panel). The bands are plotted with character in order to show the strong hybridization between $\mathrm{Cr}$ and $\mathrm{O}$ close to the Fermi energy. Red means mainly $d \mathrm{Cr}$ character, and blue means $p \mathrm{O}$ character. Also shown are the DOSs projected on each nonequivalent $\mathrm{O}$ atom and each $\mathrm{Cr}$ orbital. The two horizontal dashed lines delimit the energy window where the Wannierization process takes place.

representation of the electronic band structure of a system in an energy region of interest, which defines a Hilbert subspace. Then, the Hamiltonian expressed in the base of MLWFs includes de effect can be mapped to a tight-binding-based model which describes the system in the target Hilbert subspace. These MLWFs can be derived from Bloch states of a DFT calculation by the so-called Wannierization process as implemented in the WANNIER90 code [29]. However, the input of WANNIER90 requires the overlap matrices and projections to the Hilbert subspace, so we use the WIEN2WANNIER [30] routine as an interface among them.

The target Hilbert subspace is chosen in order to describe the $\mathrm{Cr} d$ orbitals near the Fermi energy, which are responsible for the magnetic ordering in the system. In Fig. 2, the horizontal dashed lines at -1.5 and $1 \mathrm{eV}$ delineate the chosen energy window where the Wannierization process takes place. Within the selected energy window, the number of desired Wannier functions is equal to the number of $t_{2 g}$ orbitals multiplied by the number of $\mathrm{Cr}$ atoms in the unit cell, which in our case is 12 ( $3 t_{2 g}$ orbitals times $4 \mathrm{Cr}$ atoms per unit cell). Also, we have to take into account that there are 14 bands lying inside the chosen energy window, meaning that the disentanglement procedure must be used before the Wannierization procedure takes place. Nevertheless, fast and accurate Wannierization can be done using as an initial guess the expected $t_{2 g}$-like wave functions. Our results fulfill the convergence criteria established in Ref. [29]. The tight-binding fit, if included in Fig. 2, would be indistinguishable from the DFT results, and therefore, we omit it for the sake of clarity

The obtained MLWFs confirm the expected $t_{2 g}$-type symmetry and are centered at $\mathrm{Cr}$ atoms, as shown in Fig. 3. Nevertheless, the isosurface plot in the real space of the MLWFs reveals the strong hybridization between $\mathrm{Cr}$ and $\mathrm{O}$ orbitals, in agreement with the projected density of states shown in Fig. 2. The amount of covalency that the MLWFs represents can be estimated by integrating the projected den-

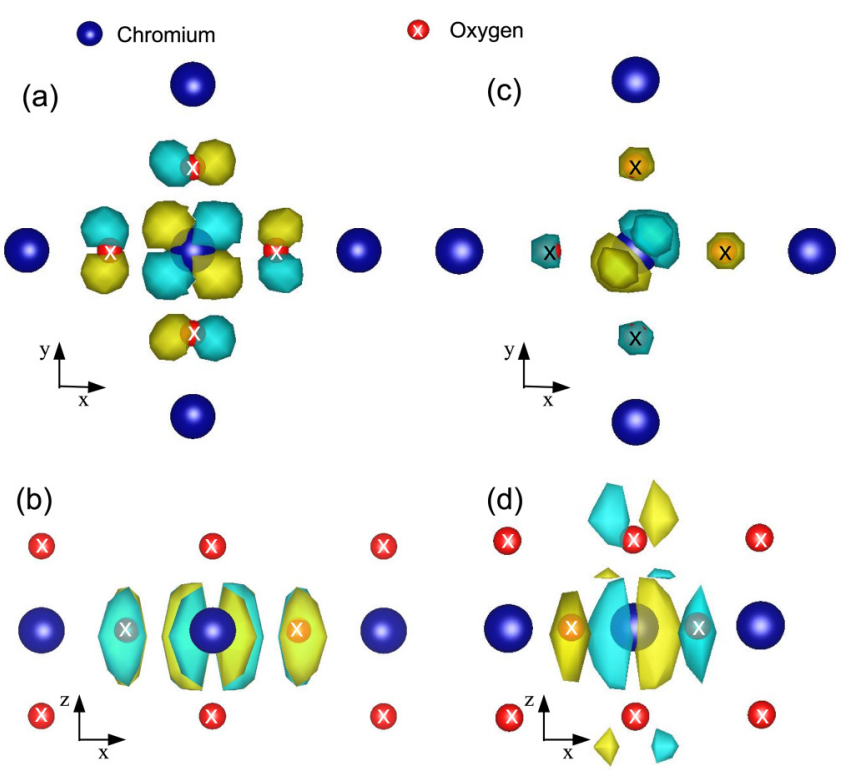

FIG. 3. Isosurface plot of two maximally localized Wannier wave functions centered at the $\mathrm{Cr}$ atom (blue circle). (a) and (b) Two views of the wave function with $x y$ symmetry, showing strong hybridization with in plane oxygens $\left(\mathrm{O}_{1}\right)$. (c) and (d) Two views of the wave function with symmetry near $x z+y z$. Note in (d) the different hybridizations between oxygen on top of chromium $\left(\mathrm{O}_{2}\right)$ and the one at the bottom $\left(\mathrm{O}_{3}\right)$. The $\mathrm{Sr}$ atoms are not shown.

sity of states of orbitals with different symmetries inside the chosen energy window where the Wannierization procedure has been performed. We find that $78.4 \%$ of the states correspond to $\mathrm{Cr} t_{2 g}$ orbitals, $2.3 \%$ correspond to $\mathrm{Cr} e_{g}$ orbitals, and $19.3 \%$ correspond to $\mathrm{O} p$ orbitals, where the majority of them $(14.6 \%)$ are $p_{x}$ and $p_{y}$ orbitals. In addition, note that the point group at the $\mathrm{Cr}$ atoms does not contain the reflection through the $\mathrm{CrO}_{2}$ layers (in particular, the distances between $\mathrm{O}_{2}$ and $\mathrm{O}_{3}$ atoms and the $\mathrm{Cr}$ atoms, $d_{2}$ and $d_{3}$ in Fig. 1, are different), a fact that is evident in the different contents of $\mathrm{O}_{2}$ and $\mathrm{O}_{3} p$ orbitals in the Wannier functions with approximate $x z$ and $y z$ symmetries. The aforementioned facts allow us to conclude that the hoppings obtained from the Hamiltonian in the base of MLWFs includes de effect of $\mathrm{O}$ atoms in the effective $\mathrm{Cr}-\mathrm{Cr}$ hopping processes.

This suggests that a simplified multiband model can be proposed (as we do in the following section) without the need to include crossed terms between $d$ and $p$ orbitals.

\section{THE MULTIBAND HUBBARD MODEL}

The multiband model is constructed from effective $t_{2 g}$ orbitals at each $\mathrm{Cr}$ site, the difference between on-site energies and the effective hopping between orbitals at different sites calculated with DFT + MLWFs, and the interactions between electrons at the same site. The Hamiltonian is

$$
H_{m}=\sum_{i, \mathbf{r}}\left(H_{C F}^{i \mathbf{r}}+H_{I}^{i \mathbf{r}}\right)+H_{h} .
$$

Here $i=1,2$ indicates the upper or lower square sublattice at positions $z= \pm 3.87 \AA / 2$, and $\mathbf{r}=a(n \hat{\mathbf{x}}+m \hat{\mathbf{y}})$, with 
$a=3.796 \AA$ and $n, m$ being integers, denotes the position of a $\mathrm{Cr}$ atom within the plane. The creation operators of effective $t_{2 g}$ orbitals at site $i, \mathbf{r}$ with spin $\sigma$ are denoted by $d_{i \mathrm{r} \alpha \sigma}^{\dagger}$, where $\alpha=x y, x z$, or $y z$. As discussed in the previous section, these effective orbitals contain some admixture with $2 p \mathrm{O}$ orbitals (and probably other orbitals) and do not have a definite symmetry under the reflection $z \rightarrow-z$. However, for simplicity we keep the notation corresponding to $t_{2 g}$ orbitals in cubic symmetry ( $x y, x z$, or $y z)$.

The first term in Eq. (1) corresponds to the tetragonal crystal field, which raises the energy of an electron in the $d_{x y}$ orbital with respect to the other two:

$$
H_{C F}^{i \mathbf{r}}=-\delta \sum_{\sigma} n_{i \mathbf{r} x y \sigma},
$$

where $n_{i \mathbf{r} \alpha \sigma}=d_{i \mathbf{r} \alpha \sigma}^{\dagger} d_{i \mathbf{r} \alpha \sigma}$. The second term of $H_{m}$ contains the on-site interactions and takes the form $[9,26,27]$

$$
\begin{aligned}
H_{I}^{i \mathbf{r}}= & U \sum_{\alpha} n_{i \mathbf{r} \alpha \uparrow} n_{i \mathbf{r} \alpha \downarrow}+\frac{1}{2} \sum_{\alpha \neq \beta, \sigma \sigma^{\prime}}\left(U^{\prime} n_{i \mathbf{r} \alpha \sigma} n_{i \mathbf{r} \beta \sigma^{\prime}}\right. \\
& \left.+J d_{i \mathbf{r} \alpha \sigma}^{\dagger} d_{i \mathbf{r} \beta \sigma^{\prime}}^{\dagger} d_{i \mathbf{r} \alpha \sigma^{\prime}} d_{i \mathbf{r} \beta \sigma}\right) \\
& +J \sum_{\alpha \neq \beta} d_{i \mathbf{r} \alpha \uparrow}^{\dagger} d_{i \mathbf{r} \alpha \downarrow}^{\dagger} d_{i \mathbf{r} \beta \downarrow} d_{i \mathbf{r} \beta \uparrow .}
\end{aligned}
$$

In the following we will take $U^{\prime}=U-2 J$, which corresponds to spherical symmetry [26] (like for the free atom).

The hopping between $t_{2 g}$ orbitals is mediated by $\mathrm{Cr}-\mathrm{O}$ hopping through $\mathrm{O} 2 p$ orbitals, and the symmetry of the orbitals imposes restrictions on the allowed processes. As a consequence, the $x y$ orbitals cannot hop in the $z$ direction. Similarly, the $x z(y z)$ orbitals cannot hop in the $y(x)$ direction. Then, the hopping term of the multiband model has the form

$$
\begin{aligned}
H_{h}= & {\left[-t_{z} \sum_{\mathbf{r}} \sum_{\alpha \neq x y} d_{1 \mathbf{r} \alpha \sigma}^{\dagger} d_{2 \mathbf{r} \alpha \sigma}-t_{x y} \sum_{i, \mathbf{r}} d_{i \mathbf{r}+\mathbf{a}, x y \sigma}^{\dagger} d_{i \mathbf{r}, x y \sigma}\right.} \\
& -t_{p} \sum_{i, \mathbf{r}}\left(d_{i \mathbf{r}+\mathbf{a} \hat{\mathbf{x}}, x z \sigma}^{\dagger} d_{i \mathbf{r}, x z \sigma}+d_{i \mathbf{r}+a \hat{\mathbf{y}}, y z \sigma}^{\dagger} d_{i \mathbf{r}, y z \sigma}\right) \\
& + \text { H.c. }],
\end{aligned}
$$

where $\mathbf{a}$ is a vector connecting two nearest $\mathrm{Cr}$ atoms in the $+x$ or $+y$ direction.

The crystal-field splitting $\delta$ and the hopping parameters $t_{z}$, $t_{p}$ and $t_{x y}$ were determined from the MLWFs, as described in Section III.

The resulting crystal-field splitting is $\delta=0.040 \mathrm{eV}$. This is likely an underestimation because orbital polarization, which is not properly taken into account by DFT, leads to larger splittings [26]. In any case, its detailed value affects very little our results and does not change our conclusions as long as $\delta>0$. A negative $\delta$ would lead to a trivial pseudospin singlet configuration $d_{x z}^{1} d_{y z}^{1}$ at each site and is inconsistent with a change in entropy near $R \ln (6)$ observed in the magnetic transition [21].

The resulting hopping parameters are $t_{z}=0.235 \mathrm{eV}$, $t_{p}=0.214 \mathrm{eV}$, and $t_{x y}=0.248 \mathrm{eV}$. They are of the same order of magnitude. The fact that $t_{x y}>t_{p}$ is expected in perturbation theory in the $\mathrm{Cr}-\mathrm{O}$ hopping because in the expression for $t_{x y}$, the denominator involves the energy necessary to take an electron from the $\mathrm{O}$ atom and put it in an $x y$ orbital, and this is smaller than the corresponding denominator for $t_{p}$. Instead, the fact that $t_{z}>t_{p}$ is rather unexpected because the interlayer distance is almost $2 \%$ larger than $a$, the shortest distance between $\mathrm{Cr}$ atoms in the plane. However, different on-site energies of the $\mathrm{O}$ orbitals lying in between the $\mathrm{Cr}$ atoms and the fact that the effective orbitals are deformed with respect to the ideal shape (as evidenced in Fig. 3) can modify the result. The deformation of the orbitals can also explain an effective hopping between $x z(y z)$ orbitals in the $y(x)$ direction with a magnitude of $0.035 \mathrm{eV}$ absent in perturbation theory in the Cr-O hopping because of symmetry. We neglect this contribution.

Concerning the values of the interactions $U$ and $J$, a fit of the lowest-atomic-energy levels along the $3 d$ series gives $J=0.70 \mathrm{eV}$ [31]. We take this value as a basis for our study. Since $J$ does not involve charge transfer, it is usually not screened in the solids, in contrast to $U$. However, in our case since the effective $t_{2 g}$ orbitals contain some orbital admixture, $J$ can be smaller. A reasonable value for $U$ for early transition metals, already used to study the orbital Kondo effect in V-doped $1 T$ - $\mathrm{CrSe}_{2}$ [17], is $\sim 4 \mathrm{eV}$. We shall analyze the dependence of the results on $U$.

\section{THE KUGEL-KHOMSKII HAMILTONIAN}

For two electrons per site and large enough $U$ the system described by the multiband Hamiltonian (1) leads to an insulating ground state, in agreement with the experimental evidence in $\mathrm{Sr}_{3} \mathrm{Cr}_{2} \mathrm{O}_{7}$ [21]. In this case, the hopping term $H_{h}$ can be eliminated from $H_{m}$ by means of a canonical transformation (similar to the derivation of the Heisenberg model from the Hubbard one [32]), leading to an effective Kugel-Khomskii Hamiltonian for the spin-1 and pseudospin$1 / 2$ (orbital) degrees of freedom. The eigenstates and corresponding energies of $H_{m}-H_{h}$ that we need [see Eqs. (1), (2), and (3)] were calculated in Ref. [9]. We restrict ourselves to second order in $H_{h}$ and denote the spin at each site by $\mathbf{S}_{i \mathrm{r}}$ and the pseudospin by $\mathbf{T}_{i \mathbf{r}}$, with $T_{i \mathbf{r}}^{z}=-1 / 2(1 / 2)$ corresponding to the $d_{x y}^{1} d_{x z}^{1}\left(d_{x y}^{1} d_{y z}^{1}\right)$ configuration. The resulting effective Hamiltonian can be written as

$$
H_{\mathrm{KK}}=\sum_{\mathbf{r}} H_{\mathbf{r}}^{\perp}+\sum_{i} H_{i}^{p},
$$

where $H_{\mathbf{r}}^{\perp}$ contains the vertical interactions (in the $z$ direction) for each two-dimensional position $\mathbf{r}$ in the $x y$ plane and $H_{i}^{p}$ describes the interactions in plane $i=1,2$. Dropping irrelevant constants, one has

$$
\begin{aligned}
H_{\mathbf{r}}^{\perp}= & \frac{I_{S}}{4} \mathbf{S}_{1 \mathbf{r}} \cdot \mathbf{S}_{2 \mathbf{r}}+I_{T} \mathbf{T}_{1 \mathbf{r}} \cdot \mathbf{T}_{2 \mathbf{r}} \\
& +I_{S T}\left(\mathbf{S}_{1 \mathbf{r}} \cdot \mathbf{S}_{2 \mathbf{r}}\right)\left(\mathbf{T}_{1 \mathbf{r}} \cdot \mathbf{T}_{2 \mathbf{r}}\right),
\end{aligned}
$$

where the factor of $1 / 4$ in the first term is introduced to compensate for factors of $\pm 1 / 4$ that come from $\mathbf{T}_{1 \mathbf{r}} \cdot \mathbf{T}_{2 \mathbf{r}}$ in classical orderings and renders the qualitative discussion 
below easier. The coefficients are

$$
\begin{aligned}
\frac{I_{S}}{t_{z}^{2}} & =-\frac{4}{3 U_{0}}+\frac{7}{3 U_{3}}+\frac{1}{U_{5}}, \\
\frac{I_{T}}{t_{z}^{2}} & =\frac{8}{3 U_{0}}+\frac{1}{3 U_{3}}-\frac{1}{U_{5}}, \\
\frac{I_{S T}}{t_{z}^{2}} & =\frac{4}{3 U_{0}}-\frac{1}{3 U_{3}}+\frac{1}{U_{5}},
\end{aligned}
$$

with

$$
\begin{aligned}
& U_{n}=U_{0}+n J \\
& U_{0}=U-3 J
\end{aligned}
$$

$U_{0}$ is the energy necessary to take a $d_{x z}\left(d_{y z}\right)$ electron from the ground state of the $d_{x y}^{1} d_{x z}^{1}\left(d_{x y}^{1} d_{y z}^{1}\right)$ configuration and add it to the $d_{x y}^{1} d_{y z}^{1}\left(d_{x y}^{1} d_{x z}^{1}\right)$ configuration of a neighboring site to build the ground state of the $d_{x y}^{1} d_{x z}^{1} d_{y z}^{1}$ configuration.

Similarly, for the interactions in each plane

$$
\begin{aligned}
H_{i}^{p}= & \sum_{\mathbf{r a}}\left[\frac{I_{S}^{p}}{4} \mathbf{S}_{i \mathbf{r}} \cdot \mathbf{S}_{i \mathbf{r}+\mathbf{a}}+I_{T}^{p} T_{i \mathbf{r}}^{z} T_{i \mathbf{r}+\mathbf{a}}^{z}\right. \\
& \left.+I_{S T}^{p}\left(\mathbf{S}_{j} \cdot \mathbf{S}_{j+\mathbf{a}}\right) T_{i \mathbf{r}}^{z} T_{i \mathbf{r}+\mathbf{a}}^{z}\right] \\
& +I_{A} \sum_{\mathbf{r}}\left[-\left(\mathbf{S}_{i \mathbf{r}} \cdot \mathbf{S}_{i \mathbf{r}+a \hat{\mathbf{x}}}\right)\left(T_{i \mathbf{r}}^{z}+T_{i \mathbf{r}+a \hat{\mathbf{x}}}^{z}\right)\right. \\
& \left.+\left(\mathbf{S}_{i \mathbf{r}} \cdot \mathbf{S}_{i \mathbf{r}+a \hat{\mathbf{y}}}\right)\left(T_{i \mathbf{r}}^{z}+T_{i \mathbf{r}+a \hat{\mathbf{y}}}^{z}\right)\right],
\end{aligned}
$$

where

$$
\begin{aligned}
I_{S}^{p} & =I_{x y}+t_{p}^{2}\left(-\frac{2}{3 U_{0}}+\frac{7}{6 U_{3}}+\frac{1}{2 U_{5}}\right), \\
\frac{I_{x y}}{t_{x y}^{2}} & =2\left(\frac{1+\delta / r}{U_{4}-r}+\frac{1-\delta / r}{U_{4}+r}\right), r=\sqrt{\delta^{2}+J^{2}}, \\
\frac{I_{T}^{p}}{t_{p}^{2}} & =\frac{4}{3 U_{0}}+\frac{1}{6 U_{3}}-\frac{1}{2 U_{5}}, \\
\frac{I_{S T}^{p}}{t_{p}^{2}} & =\frac{2}{3 U_{0}}-\frac{1}{6 U_{3}}+\frac{1}{2 U_{5}}, \\
\frac{I_{A}}{t_{p}^{2}} & =\frac{1}{2 U_{3}}+\frac{1}{2 U_{5}} .
\end{aligned}
$$

At this point we discuss qualitatively the meaning of $H_{\mathrm{KK}}$ and the expected physics. We begin discussing the two-site vertical interactions $H_{\mathbf{r}}^{\perp}$ [Eq. (6)]. For $J=0$, all interactions are equal [see Eq. (7)]: $I_{S}=I_{T}=I_{S T}=I=$ $2 t_{z}^{2} / U_{0}$. This means that without the spin-pseudospin interaction $I_{S T}$ both spins and pseudospins minimize the energy for an antiferromagnetic (AF) alignment, but the term in $I_{S T}$ is minimized for one ferromagnetic (FM) alignment and a second $\mathrm{AF}$ alignment. As a consequence, from the four classical possibilities of orienting the spin and pseudospin as FM or AF, all of them are part of the degenerate ground state with energy $-I / 2$ except for the FMFM one. This result is easy to understand: the secondorder correction to the energy of these states contains virtual processes in which one electron in the $x z$ (pseudospin $\downarrow$ ) or $y z$ (pseudospin $\uparrow$ ) orbital and spin $\uparrow$ or $\downarrow$ jumps to the other site and comes back. The corresponding gain in energy is the same for any alignment of spin and pseudospin except in the case in which the same orbital with the same spin is occupied at both sites because of the Pauli principle. If the $x y$ orbitals were absent, leaving spins $1 / 2$, this picture would not be modified by quantum fluctuations. Actually, in this case the model would have SU(4) symmetry with spin and pseudospin playing a similar role [19]. In our actual case with $S=1$, the pseudospins $1 / 2$ are more quantum than the spins 1 , and the ground state of the dimer is a pseudospin singlet and spin triplet with energy $\left(I_{S}-3 I_{T}-3 I_{S T}\right) / 4=-5 I / 4$. The first excited state is a pseudospin triplet and spin singlet with energy $\left(-2 I_{S}+I_{T}-2 I_{S T}\right) / 4=-3 I / 4$.

When $J$ (the interaction responsible for the Hund rules) is increased, as expected, the ferromagnetic spin interactions are favored. From Eqs. (7) it is apparent that $I_{S}$ decreases more strongly than the other two, clearly favoring the pseudospin singlet and spin triplet. A disadvantage of the pseudospin singlet is that it cannot take advantage of the pseudospin interactions in the plane (except for some fluctuations).

Leaving aside for the moment the contribution $I_{x y}$ due to the hopping of the $d_{x y}$ orbitals, the interactions in the plane $I_{S}^{p}$, $I_{T}^{p}$, and $I_{S T}^{p}$ are exactly half of the corresponding ones in the vertical direction if $t_{p}=t_{z}$. This factor is due to the fact that for a given direction in the plane, only one of the degenerate $x z$ and $y z$ orbitals can hop. The anisotropy in direction is reflected by the term proportional to $I_{A}$. Another consequence of the fact that $x z(y z)$ orbitals can hop only in the $x(y)$ direction in the plane is the absence of pseudospin flip terms in $H_{k}$ [see Eq. (9)].

For $t_{x y}=J=0$ one has $I_{S}^{p}=I_{T}^{p}=I_{S T}^{p}=I_{A}=t_{p}^{2} / U_{0}$. From the influence of $J$ on the parameters (similar to the case of the vertical interaction) one would expect AF pseudospin ordering and FM spin ordering to be favored. However the contribution due to the hopping of the $d_{x y}$ orbitals dominates the spin ordering. For $J=0, I_{x y}=4 t_{p}^{2} /\left(U_{0}-\delta\right)$. The prefactor 4 with respect to the other interactions in the plane is due to a factor of 2 because the $d_{x y}$ orbitals can hop in both the $x$ and $y$ directions and another factor of 2 because the FM spin alignment cannot gain energy even for $\mathrm{AF}$ pseudospin ordering. For hoppings of the same order of magnitude, clearly, $I_{x y}$ dominates over the other interactions, and one expects AF spin ordering within the planes. From the argument given above, one expects in addition FM spin ordering between planes, in agreement with the spin ordering observed by neutron scattering and calculated with $a b$ initio methods [21].

Concerning pseudospin ordering, from the values of $I_{T}$ and $I_{T}^{p}$ given above and results in the literature $[23,24]$ for $S=0$ (neglecting spins) one would expect the quantum phase transition between an AF Neel ordered phase and the phase with vertical singlet dimers to take place for $t_{z}^{2} / t_{p}^{2} \approx 2.522 / 2$ or $t_{z} / t_{p}=1.123$. However, for the actual spin $S=1$ of the system, the observed spin ordering and the effect of the interactions between spins and pseudospins $I_{S T}$ and $I_{S T}^{p}$ favor singlet ordering between planes and weaken the effective intraplane $\mathrm{AF}$ pseudospin interaction.

Taking $J=0.7 \mathrm{eV}, U=4.1 \mathrm{eV}$, which implies $U_{0}=2 \mathrm{eV}$, leads to the values tabulated in Table I for the parameters of $H_{\mathrm{KK}}$. 
TABLE I. Parameters of $H_{\mathrm{KK}}$ (in meV) for $U=4.1 \mathrm{eV}, J=$ $0.7 \mathrm{eV}$, and other parameters determined by the $a b$ initio calculations

\begin{tabular}{lcccccc}
\hline \hline$I_{S}$ & $I_{T}$ & $I_{S T}$ & $I_{S}^{p}$ & $I_{T}^{p}$ & $I_{S T}^{p}$ & $I_{A}$ \\
\hline 4.2 & 68.1 & 42.4 & 54.7 & 28.2 & 17.6 & 9.7 \\
\hline \hline
\end{tabular}

From the results (rather expected from the above discussion), it is clear that the dominant vertical interaction is $I_{T}$, which favors pseudospin singlets or possibly AF vertical order. This, together with the effect of $I_{S T}$, which is about ten times larger than $I_{S}$, overcomes the weak antiferromagnetic interaction $I_{S}$, and FM vertical spin alignment is clearly favored. Instead, in the planes the dominant interaction is $I_{S}^{p}$, which favors spin $\mathrm{AF}$ order. In addition the interaction between spins and pseudospins $I_{S T}^{p}$ is smaller than $I_{T}^{p}$, and therefore, AF orbital order in the plane is also expected. Finally, the anisotropic interaction $I_{A}$, which favors FM orbital order, is clearly smaller and has no relevant effect.

It is interesting to note that for the related insulating compound $\mathrm{BaCrO}_{3}$, calculations using DFT and dynamical mean-field theory lead to AF spin and orbital ordering in the $\mathrm{CrO}_{2}$ planes [20].

A detailed study of the competition between vertical pseudospin singlets and long-range pseudospin $\mathrm{AF}$ ordering is the subject of the following section.

\section{THE SPIN AND PSEUDOSPIN ORDERING}

In this section, we report on our study of the stability of two phases, I and II, which are the most likely according to the analysis of the previous section and a numerical study on a small cluster [21]. In both of them, the spin ordering corresponds to the experimentally observed one: antiferromagnetic in the planes and ferromagnetic between planes. In phase I, the pseudospins form vertical dimer singlets, and in phase II they order antiferromagnetically in both directions.

To calculate the energy and stability of phase I, we used the idea of the bond-operator formalism [33-37], but in the form of a generalized spin-wave theory [38], which allows us to avoid the use of Lagrange multipliers. For phase II we use ordinary spin-wave theory.

The vertical pseudospin singlet $(|\uparrow \downarrow\rangle-|\downarrow \uparrow\rangle) / \sqrt{2}$, where the first arrow denotes $T_{1 \mathrm{r}}^{z}$, can be represented using a boson operator $s_{\mathbf{r}}^{\dagger}$ as $s_{\mathbf{r}}^{\dagger}|0\rangle$, where $|0\rangle$ represents the boson vacuum. The interplane term in the Hamiltonian mixes this state with the triplet with projection 0 , which can be represented as $t_{\mathbf{r}}^{\dagger}|0\rangle=(|\uparrow \downarrow\rangle+|\downarrow \uparrow\rangle) / \sqrt{2}$, because $T_{1 \mathbf{r}}^{z} s_{\mathbf{r}}^{\dagger}|0\rangle=t_{\mathbf{r}}^{\dagger}|0\rangle / 2$, $T_{2 \mathbf{r}}^{z} s_{\mathbf{r}}^{\dagger}|0\rangle=-t_{\mathbf{r}}^{\dagger}|0\rangle / 2$, and the same interchanging $s_{\mathbf{r}}$ and $t_{\mathbf{r}}$. Following Ref. [38], we assume for phase I that the number of triplet excitations is small and "condense" the singlets using

$$
s_{\mathbf{r}}^{\dagger}=s_{\mathbf{r}}=\sqrt{1-t_{\mathbf{r}}^{\dagger} t_{\mathbf{r}}} .
$$

For the spins we use the usual Holstein-Primakoff bosons, proceeding in a similar way [38]. Performing a rotation of the spins in half of the sites by $\pi$ around the $x$ axis to convert the $\mathrm{AF}$ order in the plane in a translationally invariant FM order [39] and retaining as usual terms up to quadratic in the bosonic operators, the Hamiltonian $H_{\mathrm{KK}}$ [see Eqs. (5), (6), and (9)] for phase I becomes

$$
\begin{aligned}
H_{\mathrm{KK}}(\mathrm{I}) \simeq & N\left[I_{S}-3\left(I_{T}+I_{S T}\right)-4 I_{S}^{p}\right] / 4 \\
& +\sum_{\mathbf{r}}\left(I_{T}+I_{S T}\right) t_{\mathbf{r}}^{\dagger} t_{\mathbf{r}} \\
& +\sum_{\mathbf{r}, \mathbf{a}}\left(\frac{I_{T}^{p}-I_{S T}^{p}}{2}\right)\left(t_{\mathbf{r}}^{\dagger} t_{\mathbf{r}+\mathbf{a}}^{\dagger}+\text { H.c. }\right) \\
& +\sum_{i, \mathbf{r}}\left(\frac{-I_{S}+3 I_{S T}+4 I_{S}^{p}}{4}\right) b_{i \mathbf{r}}^{\dagger} b_{i \mathbf{r}} \\
& +\sum_{\mathbf{r}}\left(\frac{I_{S}-3 I_{S T}}{4}\right)\left(b_{1 \mathbf{r}}^{\dagger} b_{2 \mathbf{r}}+\text { H.c. }\right) \\
& \times \sum_{i, \mathbf{r}, \mathbf{a}} \frac{I_{S}^{p}}{4}\left(b_{i \mathbf{r}}^{\dagger} b_{i \mathbf{r}+\mathbf{a}}^{\dagger}+\text { H.c. }\right),
\end{aligned}
$$

where $N$ is the number of sites in a plane and $b_{i \mathbf{r}}^{\dagger}$ creates a spin excitation at the two-dimensional position $\mathbf{r}$ of plane $i$.

Diagonalizing the Hamiltonian by means of a standard Bogoliubov transformation, the ground-state energy becomes

$$
\begin{aligned}
E(\mathrm{I}) \simeq & N\left[I_{S}-3\left(I_{T}+I_{S T}\right)-4 I_{S}^{p}\right] / 4 \\
& +\sum_{k, j=1}^{3} \frac{\lambda_{k}^{j}-A_{j}}{2}
\end{aligned}
$$

with

$$
\begin{aligned}
& \lambda_{k}^{j}=\sqrt{A_{j}^{2}-B_{j}^{2}\left[\cos \left(k_{x} a\right)+\cos \left(k_{y} a\right)\right]^{2}}, \\
& A_{1}=I_{T}+I_{S T}, B_{1}=I_{T}^{p}-I_{S T}^{p}, \\
& A_{2}=I_{S}^{p}, \\
& A_{3}=\frac{-I_{S}+3 I_{S T}+2 I_{S}^{p}}{2}, \\
& B_{2}=B_{3}=I_{S}^{p} / 2 .
\end{aligned}
$$

Note that when $2 B_{1}>A_{1}$, the system becomes unstable against the creation of triplet excitations of long wavelength $k_{x}, k_{y} \rightarrow 0$ and Eq. (13) becomes meaningless. In general, if for some parameters the assumed pseudospin or spin arrangements become unstable, the situation is detected in the numerical algorithm used to calculate the two-dimensional integral over $\left(k_{x}, k_{y}\right)$ by the nonanalyticity of some expression for small $\left(k_{x}, k_{y}\right)$. In fact, as we show below, phase I becomes unstable near the transition to phase II (as might be expected).

For phase II with long-range spin and pseudospin ordering, a treatment similar to that above using Holstein-Primakoff bosons leads to the following energy:

$$
\begin{aligned}
E(\mathrm{II}) \simeq & N\left[\left(I_{S}-I_{T}-I_{S T}\right) / 4-I_{T}^{p}-I_{S}^{p}+I_{T S}^{p}\right] \\
& +\sum_{k, j=4}^{5} \frac{\mu_{k}^{j}-A_{j}}{2}+\sum_{k, j=6}^{7} \frac{\lambda_{k}^{j}-A_{j}}{2},
\end{aligned}
$$

where

$$
\begin{aligned}
& A_{4}=A_{5}=\frac{I_{T}+I_{S T}}{2}+2 I_{T}^{p}-2 I_{T S}^{p}, \\
& \mu_{k}^{j}=\sqrt{A_{j}^{2}-C_{j}^{2}},
\end{aligned}
$$




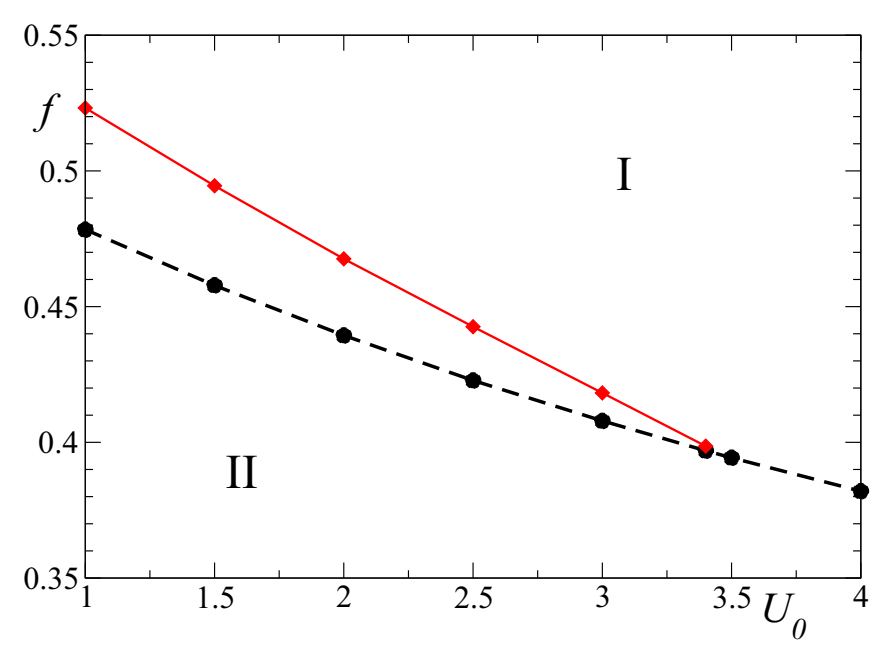

FIG. 4. Factor in which the vertical hopping $t_{z}$ has to be reduced to destabilize the dimerized pseudospin singlet phase $\mathrm{I}$ for $J=$ $0.7 \mathrm{eV}$. The solid line denotes the crossing of the energies $E(\mathrm{II})=$ $E(\mathrm{I})$, and the dashed line is the limit of the stability of phase I (see text).

$$
\begin{aligned}
C_{4(5)}= & \left(I_{T}^{p}-I_{T S}^{p}\right)\left[\cos \left(k_{x} a\right)+\cos \left(y_{x} a\right)\right] \\
& +(-) \frac{I_{T}+I_{S T}}{2} \\
A_{6(7)}= & \frac{-I_{S}+I_{S T}}{4}+I_{S}^{p}-I_{S T}^{p} \\
& +(-) \frac{I_{S}-I_{S T}}{4} \\
B_{6}= & B_{7}=\frac{I_{S}^{p}-I_{T S}^{p}}{2} .
\end{aligned}
$$

As a test of our procedure we have compared the energy of the two phases when all interactions involving spin are zero (this is equivalent to taking $S=0$ ), leaving only $I_{T}$ and $I_{T}^{p}$. We obtain a transition between the long-range ordered phase II for small $I_{T}$ to the phase of vertical dimers I for large $I_{T}$ at $I_{T} / I_{T}^{p}=2.947,17 \%$ larger than the value near 2.522 obtained by Monte Carlo calculations [23,24]. Thus, our approach underestimates the stability of phase I.

For the parameters listed in Table I, we find that the energy of the dimerized phase I is lower than the long-range-ordered phase II by $19.8 \mathrm{meV}$. This agrees with the structural measurements, which do not detect any distortion of the lattice or displacement of the $\mathrm{O}$ atoms expected for long-range orbital ordering. As a test of the stability of this phase, we have lowered the vertical hopping $t_{z}$ by a factor $f$ and searched for the value of $f$ that leads to the equality of both energies $[E(\mathrm{II})=E(\mathrm{I})]$ for different values of $U$. The results are shown by the solid line in Fig. 4. For the expected value of $U \sim 4.1 \mathrm{eV}\left(U_{0}=U-3 \mathrm{~J} \sim 2 \mathrm{eV}\right)$, the resulting value of $f$ is slightly larger than the value of $f$ that corresponds to the instability of the dimerized phase against the formation of triplet excitations [given by $A_{1}=2 B_{1}$; see the discussion after Eq. (13)], corresponding to the dashed line in Fig. 4. Both values of $f$ are of the order of 0.5 , reflecting the fact that the real system is far from the boundary of the phase diagram. For

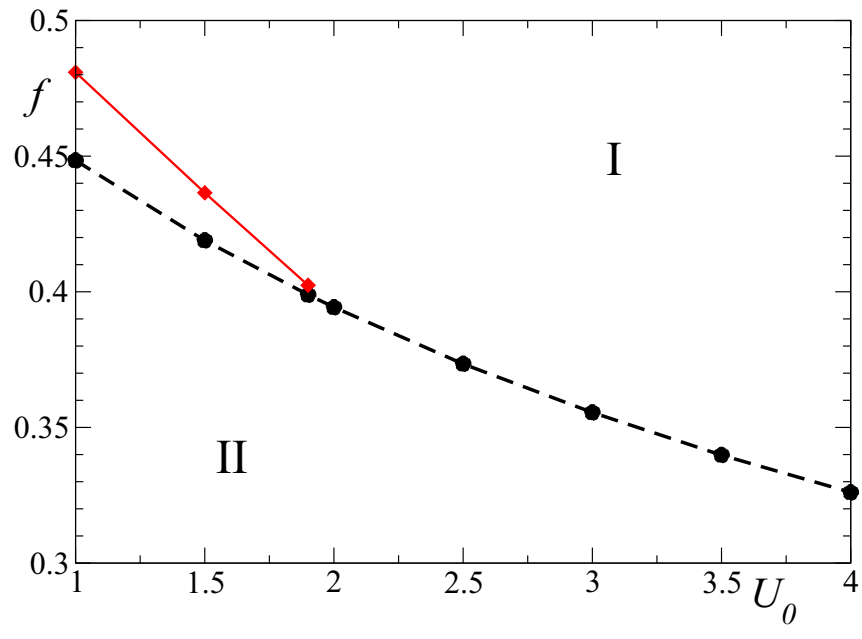

FIG. 5. Same as Fig. 4 for $J=0.4 \mathrm{eV}$.

larger values of $U$, the dimerized phase is stabilized further. For $U_{0}>3.5 \mathrm{eV}(U>5.6)$, the dimerized phase I becomes unstable at a point at which its energy is still lower than that of the long-range-ordered one II. This is probably a shortcoming of the approximations. From the physics of the case of spin $S=0[23,24]$ and its extension to two cubic sublattices [13], one would expect a second-order transition between both phases and a coincidence of both transitions (solid and dashed lines).

In Fig. 5 we show how the previous results change when $J$ is reduced from 0.7 to $0.4 \mathrm{eV}$. We consider that this value is a lower bound of the interaction responsible of the Hund rules due to the fact that the effective $t_{2 g}$ orbitals are not pure $\mathrm{Cr}$ ones but have some admixture of neighboring $\mathrm{O}$ atoms with smaller interactions. In particular, from the $\mathrm{O}$ content estimated at the end of Sec. III and the fact that $\mathrm{O}$ sites with two $2 p$ holes are very rare, we estimate $J=0.57 \mathrm{eV}$.

As one can see, the changes with respect to Fig. 4 are minor. We conclude that for the calculated values of the hopping terms obtained as described in Sec. III and reasonable values of the interactions, the dimerized phase I is the stable one.

\section{SUMMARY AND DISCUSSION}

Using maximally localized Wannier functions in bilayer $\mathrm{Sr}_{3} \mathrm{Cr}_{2} \mathrm{O}_{7}$, we have derived a six-band Hubbard model for effective $\mathrm{Cr} t_{2 g}$ orbitals (three per $\mathrm{Cr}$ site) which contain some admixture with neighboring $\mathrm{O}$ atoms, estimated to be nearly $19 \%$, as discussed in Sec. III.

Using the resulting hopping and on-site energy parameters and reasonable values of the Coulomb interaction $U$ and interaction responsible for the Hund rules $J$, we have derived an effective Kugel-Khomskii Hamiltonian $H_{\mathrm{KK}}$ using a procedure equivalent to degenerate second-order perturbation theory in the hopping terms.

A similar $H_{\mathrm{KK}}$ was derived in Ref. [21] using fourth-order perturbation theory in the $\mathrm{Cr}-\mathrm{O}$ hopping, but the parameters were not determined, and it was not clear where the system lies in the phase diagram, although the absence of observable distortions is consistent with same phase that we obtain here. 
Our analysis of $H_{\mathrm{KK}}$ and calculations based on bond-order operators and spin waves show that the ground state of the system has long-range spin order, with antiferromagnetic order in the layers and ferromagnetic order between layers, in agreement with experiment and $a b$ initio calculations [21]. Instead, the orbital degrees of freedom form singlet dimers perpendicular to the planes. This rather exotic arrangement of the orbital degrees of freedom is rare. For interplane and intraplane interactions of the same order of magnitude, one expects long-range antiferromagnetic ordering of the pseudospin (orbital) degrees of freedom. Although the methods used to solve $H_{\mathrm{KK}}$ are semiquantitative, with errors of the order of $17 \%$ for a known case, the obtained ground state is rather far from the phase boundary to the phase of long-range antiferromagnetic pseudospin ordering.

The reason for the stability of the dimerized phase is twofold. On the one hand, the intraplane pseudospin interac- tions are smaller due to restrictions of the $x z$ and $y z$ orbitals to hop in certain directions of the plane. On the other hand, for the spin ordering observed, the interactions between spins and pseudospins strengthen (weaken) the antiferromagnetic pseudospin correlations normal to (in the) layers. This aspect has some similarities to the physics of some $\mathrm{V}$ oxides, for which calculations suggest that ferromagnetic couplings are particularly strong due to singlet orbital fluctuations [14].

\section{ACKNOWLEDGMENTS}

A.A.A. (C.H.) thanks B. Normand and C. Batista (R. Weht and A. M. Llois) for helpful discussions. A.A.A. (C.H.) is sponsored by Grant No. PIP 112-201501-00506 of CONICET and Grant No. PICT 2013-1045 of the ANPCyT (Grant No. PICT 2014-1555 of the ANPCyT).
[1] K. I. Kugel and D. I. Khomskii, Sov. Phys. Usp. 25, 231 (1982).

[2] A. I. Liechtenstein, V. I. Anisimov, and J. Zaanen, Phys. Rev. B 52, R5467 (1995).

[3] T. Mizokawa and A. Fujimori, Phys. Rev. B 51, 12880(R) (1995).

[4] L. F. Feiner, A. M. Olés, and J. Zaanen, Phys. Rev. Lett. 78, 2799 (1997).

[5] C. D. Ling, J. E. Millburn, J. F. Mitchell, D. N. Argyriou, J. Linton, and H. N. Bordallo, Phys. Rev. B 62, 15096 (2000).

[6] C. Ulrich, G. Khaliullin, J. Sirker, M. Reehuis, M. Ohl, S. Miyasaka, Y. Tokura, and B. Keimer, Phys. Rev. Lett. 91, 257202 (2003)

[7] P. Horsch, G. Khaliullin, and A. M. Olés, Phys. Rev. Lett. 91, 257203 (2003).

[8] Z. Fang and N. Nagaosa, Phys. Rev. Lett. 93, 176404 (2004).

[9] A. A. Aligia and M. A. Gusmão, Phys. Rev. B 70, 054403 (2004).

[10] S. Sugai and K. Hirota, Phys. Rev. B 73, 020409(R) (2006).

[11] M. De Raychaudhury, E. Pavarini, and O. K. Andersen, Phys. Rev. Lett. 99, 126402 (2007).

[12] H. Manaka, Y. Miyashita, Y. Watanabe, and T. Masuda, J. Phys. Soc. Jpn. 76, 044710 (2007).

[13] B. Normand and A. M. Olés, Phys. Rev. B 78, 094427 (2008).

[14] A. M. Olés and P. Horsch, in Properties and Applications of Thermoelectric Materials, edited by V. Zlatić and A. C. Hewson, NATO Science for Peace and Security Series B: Physics and Biophysics (Springer, Dordrecht, 2009), p. 299.

[15] C. Stingl, R. S. Perry, Y. Maeno, and P. Gegenwart, Phys. Rev. Lett. 107, 026404 (2011).

[16] W. Brzezicki and A. M. Olés, J. Phys.: Conf. Ser. 391, 012085 (2012).

[17] M. Núñez, D. C. Freitas, F. Gay, J. Marcus, P. Strobel, A. A. Aligia, and M. Núñez-Regueiro, Phys. Rev. B 88, 245129 (2013).

[18] A. M. Lobos, M. A. Romero, and A. A. Aligia, Phys. Rev. B 89, 121406(R) (2014).

[19] A. M. Lobos and A. A. Aligia, J. Phys.: Conf. Ser. 568, 052002 (2014).

[20] G. Giovannetti, M. Aichhorn, and M. Capone, Phys. Rev. B 90, 245134 (2014).
[21] J. Jeanneau, P. Toulemonde, G. Remenyi, A. Sulpice, C. Colin, V. Nassif, E. Suard, E. Salas Colera, G. R. Castro, F. Gay, C. Urdaniz, R. Weht, C. Fevrier, A. Ralko, C. Lacroix, A. A. Aligia, and M. Núñez-Regueiro, Phys. Rev. Lett. 118, 207207 (2017).

[22] It is known that orbital-related Coulomb interactions are underestimated in the spin LDA [40], and empirical orbital polarization corrections [41] are frequently used to cure this problem.

[23] A. W. Sandvik and D. J. Scalapino, Phys. Rev. Lett. 72, 2777 (1994).

[24] L. Wang, K. S. D. Beach, and A. W. Sandvik, Phys. Rev. B 73, 014431 (2006).

[25] Y. Q. Qin, B. Normand, A. W. Sandvik, and Z. Y. Meng, Phys. Rev. B 92, 214401 (2015).

[26] A. A. Aligia, Phys. Rev. B 88, 075128 (2013).

[27] D. Ogura, H. Aoki, and K. Kuroki, Phys. Rev. B 96, 184513 (2017).

[28] K. Schwarz and P. Blaha, Comput. Mater. Sci. 28, 259 (2003).

[29] I. Souza, N. Marzari, and D. Vanderbilt, Phys. Rev. B 65, 035109 (2001).

[30] J. Kuneš, R. Arita, P. Wissgott, A. Toschi, H. Ikeda, and K. Held, Comput. Phys. Commun. 181, 1888 (2010).

[31] A. A. Aligia and T. Kroll, Phys. Rev. B 81, 195113 (2010).

[32] A. H. MacDonald, S. M. Girvin, and D. Yoshioka, Phys. Rev. B 37, 9753 (1988).

[33] A. V. Chubukov, JETP Lett. 49, 129 (1989).

[34] S. Sachdev and R. N. Bhatt, Phys. Rev. B 41, 9323 (1990).

[35] S. Gopalan, T. M. Rice, and M. Sigrist, Phys. Rev. B 49, 8901 (1994).

[36] M. Matsumoto, B. Normand, T. M. Rice, and M. Sigrist, Phys. Rev. B 69, 054423 (2004).

[37] B. Normand and Ch. Rüegg, Phys. Rev. B 83, 054415 (2011).

[38] R. A. Muniz, Y. Kato, and C. D. Batista, Prog. Theor. Exp. Phys. 2014, 083101 (2014).

[39] G. Martínez and P. Horsch, Phys. Rev. B 44, 317 (1991).

[40] H. Eschrig, M. Sargolzaei, K. Koepernik, and M. Richter, Europhys. Lett. 72, 611 (2005), and references therein.

[41] O. Eriksson, M. S. S. Brooks, and B. Johansson, Phys. Rev. B 41, 7311(R) (1990). 